\title{
Chlamydia trachomatis growth inhibition and restoration of LDL-receptor level in HepG2 cells treated with mevastatin
}

\author{
Yuriy K Bashmakov ${ }^{1 * \dagger}$, Nailya A Zigangirova ${ }^{1,2+}$, Yulia P Pashko², Lidia N Kapotina ${ }^{2}$, Ivan M Petyaev ${ }^{1}$
}

\begin{abstract}
Background: Perihepatitis is rare but consistently occurring extragenital manifestation of untreated Chlamydia trachomatis infection. Despite of possible liver involvement in generalized C. trachomatis infection, the ability of the pathogen to propagate in the hepatic cells and its impact on liver functions is not thoroughly investigated. The effect of mevastatin, an inhibitor of 3-hydroxy-3-methylglutaryl CoA reductase, on C. trachomatis growth in human hepatoma cell line HepG2 has been studied. Bacterial growth was assessed by immunostaining with FITC-labeled monoclonal antibody against chlamydial lipopolysaccharide and by RT-PCR for two chlamydial genetic markers (16S rRNA and euo).

Results: Chlamydial inclusion bodies were seen in approximately $50 \%$ of hepatocytes at 48 hours in the post infection period. Lysates obtained from infected hepatocytes were positive in the infective progeny test at 48 and especially in 72 hours after infection initiation. It has been shown that chlamydial infection in hepatocytes also leads to the decline of LDL-receptor mRNA which reflects infection multiplicity rate. Additions of mevastatin (1, 20 and $40 \mu \mathrm{M}) 1$ hour before inoculation restored and upregulated LDL-receptor mRNA level in a dose-dependent manner. Mevastatin treatment had no effect on internalization of chlamydial particles. However it reduced drastically the number of chlamydial 165 rRNA and euo transcripts as well as overall infection rate in HepG-2 cells. Complete eradication of infection has been seen by immunofluorescent staining at $40 \mu \mathrm{M}$ mevastatin concentration, when expression level of chlamydial $16 \mathrm{~S}$ rRNA and euo was undetectable. Lower concentration of mevastatin $(20 \mu \mathrm{M})$ promoted euo expression level and the appearance of atypically small chlamydial inclusions, while there was a noticeable reduction in the number of infected cells and 16S rRNA transcripts.
\end{abstract}

Conclusions: $C$. trachomatis can efficiently propagate in hepatocytes affecting transcription rate of some liverspecific genes. Ongoing cholesterol synthesis is essential for chlamydial growth in hepatocytes. Inhibitors of cholesterol biosynthesis can supplement conventional strategy in the management of $C$. trachomatis infection.

\section{Background}

Chlamydia trachomatis is a prevalent bacterial pathogen causing most of the cases of urogenital infections and preventable blindness in the world. Epididymitis and urethritis in men, cervical as well as the urethral inflammation in woman may lead to acute pelvic inflammatory disease and variety of other extragenital manifestations in both sexes. Among most frequent extragenital manifestations of C. trachomatis are sexually acquired

\footnotetext{
* Correspondence: YBash47926@aol.com

+ Contributed equally

'Cambridge Theranostics Ltd, Babraham Research Campus, Babraham, Cambridge, CB2 4AT, UK
}

reactive arthritis (SARA), conjunctivitis and perihepatitis [1]. In most of the cases of ophthalmological manifestations C. trachomatis can be detected and/or isolated in the eye swabs [2]. It is believed that immunological and hormonal phenotype as well as some genotype characteristics, particularly expression of human leucocyte antigen B27, predetermine the severity of extragenital manifestations caused by C. trachomatis [3]. Delayed cell-mediated immunological response is also known to play an important role in the systemic generalization of chlamydial disease [4].

However there is a growing body of evidence that $C$. trachomatis can be present and isolated from 
extragenital tissues and organs. Bacterial antigens, DNA and/or RNA can be detected in whole blood [5,6] since C. trachomatis can efficiently propagate in mononuclear cells [7] as well as in astrocytes [8], muscle cells [9] and myocardiocytes [10]. Virulent forms of C. trachomatis can be isolated from synovial exudate [11], ascitic fluid $[12,13]$, liver biopsy material [14], and respiratory secretion fluids [15]. Similar pattern of extragenital manifestations has been reported in animal experiments.

Lesions containing virulent $C$. trachomatis have been reported in lungs, liver and spleen of BALB/c mice in the post-infection period [16]. With the exception of a single report [14] there are no confirmed cases of $C$. trachomatis isolation from the human liver or any well articulated insights on the potential role of chlamydial infection in hepatobilliary pathology. However, recently shown ability of $C$. trachomatis to propagate in hepatocytes $[17,18]$ leads to many questions about possible involvement of liver in systemic chlamydial disease.

In the present paper we have investigated the infectability of $C$. trachomatis toward immortalized human hepatoma cells (HepG2 cell line) and some metabolic consequences of chlamydia propagation in the hepatic cell line. In particular, of mRNA regulation of major lipogenic genes in the host cells and effect of mevastatin, an inhibitor of 3-hydroxy-3-methyglutaryl CoA reductase (HMG-CoA reductase), in cases of chlamydial infection in HepG2 cells are reported below.

\section{Methods \\ Reagents}

All reagents were purchased from Sigma-Aldrich unless specifically mentioned otherwise. HepG2 and Hep2 cells were obtained from "European Collection of Cell Cultures" (Salisbury, UK).

\section{Cell culture and organisms}

HepG2 cells were cultured in $5 \% \mathrm{CO}_{2}$ in DMEM supplemented with 10\% Fetal Bovine Serum (FBS) and 2 $\mathrm{mM}$ glutamine. Cells were grown in 6, 24, and 96 well plates until confluence rate of $80 \%$ was reached. Addition of mevastatin at concentrations ranging from $1 \mu \mathrm{M}$ to $40 \mu \mathrm{M}$ was done 1 hour before inoculation of C. trachomatis. Strain L2/Bu434 of C. trachomatis was kindly provided by Dr. P. Saikku (University of Oulu, Finland). Chlamydial strains were initially propagated in Hep2 cells and purified by Renografin gradient centrifugation as described [19]. Chlamydial titers were determined by infecting Hep2 cells with 10-fold dilutions of thawed stock suspension. Purified elementary bodies (EB) with known titer were suspended in sucrose-phosphate-glutamic acid buffer [19] and used as inoculums for HepG2 cells.
HepG2 plates were infected with C. trachomatis at multiplicities of infection (MOI) of 1 or 2 in DMEM with $0.4 \%$ glucose without FBS and cycloheximide and centrifuged for 0.5 hour at $1500 \mathrm{~g}$. The cells were harvested for RNA analysis in 24 hours (expression of chlamydial genes) and in 48 hours (expression of eukaryotic genes and immunofluorescence analysis) after infection after the inoculation of $C$. trachomatis. Acell viability assay was conducted routinely for each group of the experiment using $2 \%$ trypan blue exclusion test. The cell monolayers with viability $>85 \%$ were used for RNA extraction and/or immunostaining. There was a significant decrease in number of viable hepatocytes during the late stage of chlamydial infection in HepG2 cells (72 hours).

\section{Immunofluoresence staining}

Infected HepG2 monolayers grown 48 hours on coverslips in 24 well plates, which were fixed with methanol. Permeabilized cells were stained by direct immunofluorescence using FITC - conjugated monoclonal antibody against chlamydial lipopolysaccharide (NearMedic Plus, $\mathrm{RF}$ ). Inclusion-containing cells were visualized using Nikon Eclipse 50 i microscope fluorescence microscope at X1350 magnification.

\section{Internalization assay}

Internalization assay has been performed as described [20]. Briefly, to visualize attachment of $C$. trachomatis to HepG2 cells, elementary bodies (EB) of C. trachomatis were added at MOI 50 to the 24 well plates with coverslips containing hepatocytes monolayers. The EB were allowed to attach in presence or absence of $40 \mu \mathrm{M}$ mevastatin for $60 \mathrm{~min}$ at $4^{\circ} \mathrm{C}$ after which the inoculum was removed, cell were washed 3 times with ice-cold PBS. To visualize attached particles, the cell monolayers were fixed in $4 \%$ paraformaldehyde for $15 \mathrm{~min}$ on ice. This regimen of fixation is believed to maintain the integrity of the plasma membrane in the host cells [20]. After fixation the cells were washed with PBS and incubated for 30 min with monoclonal chlamydial LPS-specific antibody labeled with FITC $(1 \mu \mathrm{g} / \mathrm{ml}$, NearMedic Plus, RF) for visualization of attached particles. Internalization has been studied in separate set of experiments. To allow attachment, HepG2 cells were incubated with EB of C. trachomatis in presence or absence of $40 \mu \mathrm{M}$ mevastatin for 1 hour at $4^{\circ} \mathrm{C}$ after which the inoculum was removed and the cells were washed 3 times with ice-cold PBS. The cells were transferred to $37^{\circ} \mathrm{C}$ for 1 hour to permit internalization. After fixation with $4 \%$ paraformaldehyde (15 min, room temperature) the cells were incubated for $30 \mathrm{~min}$ with the polyclonal antibody raised against EB of $C$. trachomatis (Gamaleya Institute of Microbiology and Epidemiology, Moscow, RF). This 
step was performed in order to block attachment sites of non-internalized EB. After fixation with methanol (15 min, room temperature), which allows penetration of antibody inside of the cells [20], cell monolayers were incubated for $30 \mathrm{~min}$ with $1 \mu \mathrm{g} / \mathrm{ml}$ of monoclonal FITC-conjugated antibody against $C$. trachomatis major outer membrane protein (MOMP) (NearMedic Plus, $\mathrm{RF})$. The cells were washed thoroughly with PBS and analyzed by immunofluorescent microscope.

\section{Assessment of infective progeny}

In order to assess the infective progeny accumulation in HepG2 cells after 48 hour cultivation period, HepG2 cells were harvested, frozen and thawed, as described elsewhere. Serial dilutions of lysates were inoculated onto Hep- 2 cells and centrifuged for 0.5 hour at 1500 g. The infected cells were visualized with $C$. trachomatis LPSspecific antibody in 48 hours of the post-infection period.

\section{RNA extraction and reverse transcription}

RNA was isolated from HepG2 monolayers grown on 6well plates using TRIZol (Invitrogen). Total mRNA pretreated with DNase I (DNA-free ${ }^{\mathrm{m}}$, Ambion) and quantified on the spectrophotometer NanoDrop ND-100 (ThermoFisher Scientific, Wilmington, USA) was converted into cDNA using random hexamer primers and a SuperScript III First-Strand Synthesis Kit (Invitrogen, Karlsruhe, Germany).

\section{Quantitative real-time PCR}

The mRNA levels for two different developmental genes of $C$. trachomatis were analyzed in HepG2 cells by quantitative RT-PCR using thermocycler ANK 32 (Syntol, RF). The 16S rRNA and gene encoding DNA-binding protein Euo were studied as constitutive markers of the early stage of chlamydial developmental cycle. Primers for C. trachomatis $16 \mathrm{~S}$ rRNA (sense - 5'-GGCGTATTTGGGCATCCGAGT AACG-3', antisense - 5'-TCAAATCCAGCGGGTATTA ACCGCCT-3') and C. trachomatis Euo (sense - 5'-TC CCCGACGCTCTCCTTTCA-3', antisense - 5'-CTCG TCAGGCTATCTATGTTGCT-3') were verified and used under thermal cycling conditions $-95^{\circ} \mathrm{C}$ for 10 min and 50 cycles of $95^{\circ} \mathrm{C}$ for 15 seconds, $60^{\circ} \mathrm{C}$ for 1 min and $72^{\circ} \mathrm{C}$ for 20 seconds. Serial dilutions of C. trachomatis RNA, extracted from chlamydia-infected Hep2 cells, were used as a standard for quantification of chlamydial gene expression. The results of PCR analysis for chlamydia-specific genes were normalized to mRNA values of human beta actin ( $\beta$-actin, primers: sense - 5'GCACCCAGCACAATGAAGAT-3', antisense - 5'-GC CGATCCACACGGAGTAC-3'). Among other humanspecific genes studied were major lipogenic enzymes: 3hydroxy-3-methyglutaryl CoA reductase (HMG CoA reductase, primers: sense-5'-CAAGGAGCATGCAAAGATAATCC-3' antisense -5'-GCCATTACGGTCC CACACA-3'); 3-hydroxy-3-methyglutaryl CoA synthase (HMG CoA Syn, primers: sense - 5'-GACTTGTGC ATTCAAACATAGCAA-3', antisense - 5'-GCTGTAGC AGGGAGTCTTGGTACT-3'); squalene synthase (SS, primers: sense - 5'-ATGACCATCAGTGTGGAAAAG AAG-3', antisense - 5'-CCGCAGTCTGGTTGGTAA-3'); and fatty acid synthase (FAS, primers: sense- 5 '-TC GTGGGCTACAGCATGGT-3', antisense - 5'-GCC CTCTGAAGTCGAAGAAGAA-3').

The mRNA levels for lipogenic enzymes as well as mRNAs for LDL-receptor (LDL-R, primers: sense 5'-GGCTGCGTTAATGTGACACTCT-3', antisense 5'-CTCTAGCCATGTT GCAGACTTTGT-3') and LDL-receptor related protein (LRP, primers: - 5'-CCT ACTGGACGCTGA CTTTGC-3' antisense - 5'-GGC CCCCCATGTAGAGTGT-3') in the host cells were normalized to human $\beta$-actin expression level. The mRNA expression levels in the host cells were referenced to the CT values in uninfected HepG2 cells grown at the same conditions. That reference value was taken as 1.00. Each cDNA sample was tested by PCR at least three times. All experiments were repeated at least twice. Representative sets of results are shown below.

\section{Results}

\section{C. trachomatis growth in HepG2 cells}

Immunofluorescent images of HepG2 infected cells reveal that $\mathrm{C}$. trachomatis can efficiently grow in immortalized hepatocytes cells line. Positive immunofluorescence was first apparent within 24 hours of postinfection period and did not differ in intensity at MOIs of 1 and 2. Inclusion bodies were seen in about $50 \%$ of cells at 48 hours in the post-infection period at MOI of 1. Up to $70 \%$ of the infected cells were seen at multiplicity rate of 2. Most of the immunostaining was localized throughout whole cytoplasm. However some cells had perinuclear pattern of immunofluorescence with no intranuclear inclusions seen. At 48 and especially 72 hours of the post-infection period, immunostaining was stronger with numerous inclusion bodies. Some of them were released from the ruptured cells. To determine if C. trachomatis can be cultured from HepG2 monolayers, we harvested 24 and 48 hour cultures of hepatocytes. Replication was not observed when 24 hour lysates of hepatocytes were inoculated to Hep2 cells. However the lysates obtained in 48 and especially 72 hour were positive in the infective progeny test.

\section{LDL-receptor mRNA and multiplicity of infection}

As can be seen from Table 1, 48 hour propagation of $C$. trachomatis in HepG2 cells did not affect mRNA for a major housekeeping gene - 36B4, nor mRNAs for 
Table 1 Folds and mRNA changes in HepG2 cells infected with $C$. trachomatis at different infectivity rates.

\begin{tabular}{|c|c|c|c|}
\hline \multirow[t]{2}{*}{ Parameter } & \multirow[t]{2}{*}{ Non-infected cells } & \multicolumn{2}{|c|}{ Infected cells } \\
\hline & & MOI 1 & $\mathrm{MOI} 2$ \\
\hline $36 \mathrm{~B} 4^{\mathrm{ct}}$ & 18.37 & 18.26 & 18.01 \\
\hline HMG-CoA Red & 1 & 1.31 & 0.98 \\
\hline HMG-CoA Synth & 1 & 1.06 & 0.87 \\
\hline SS & 1 & 1.21 & 0.89 \\
\hline LDL-R & 1 & 0.76 & 0.56 \\
\hline LRP & 1 & 0.87 & 0.99 \\
\hline FAS & 1 & 0.88 & 0.89 \\
\hline
\end{tabular}

HepG2 cells were set up, grown and infected with C. trachomatis in presence or absence of mevastatin as described in Methods. RNA was extracted in 48 hours after inoculation of the bacteria. RNA levels for the genes of interest were normalized to 36B4 expression level, whose CT values are represented in the upper row of the Table. All RNA values in the infected cells are referenced to non-infected control

lipogenic enzymes. However, there is dose-dependent decline in LDL-receptor mRNA, reflecting multiplicity infection level. LDL-receptor related protein mRNA remained unchanged.

\section{Mevastatin reverses LDL-receptor mRNA decline}

Inhibitors of HMG-CoA reductase are the most powerful activators of LDL receptor, whose activity on the LDL-receptor is mediated by SREBP pathway [21]. The addition of mevastatin to HepG2 cells infected with $C$. trachomatis at MOI of 1 did not affect cell viability nor mRNA levels of 36B4 (Table 2). However, LDL-receptor mRNA level was dose-dependently upregulated with the increasing concentrations of mevastatin, reaching 2 fold induction at $40 \mu \mathrm{M}$ level. This effect was even more pronounced at 72 hours of the post-infection period though cell viability was declining (results not shown). There is also dose-dependent upregulation of

Table 2 Folds and mRNA changes in C. trachomatisinfected HepG2 cells after addition of mevastatin.

\begin{tabular}{lccccc}
\hline Parameter & Non-infected cells & \multicolumn{4}{c}{ Infected cells - Addition of } \\
& & $\mathbf{0} \boldsymbol{\mu} \mathbf{M}$ & $\mathbf{1} \boldsymbol{\mu} \mathbf{M}$ & $\mathbf{2 0} \boldsymbol{\mu M}$ & $\mathbf{4 0} \boldsymbol{\mu M}$ \\
\hline 36B4 & & 17.04 & 16.94 & 16.98 & 17.01 \\
HMG-CoA Red & 16.94 & 1.06 & 1.17 & 1.7 & 1.81 \\
HMG-CoA Synth & 1 & 0.79 & 1.46 & 1.53 & 1.89 \\
SS & 1 & 0.87 & 1.27 & 1.54 & 1.73 \\
LDL-R & 1 & 0.69 & 1.38 & 1.63 & 2.08 \\
LRP & 1 & 1.09 & 0.85 & 0.91 & 0.99 \\
FAS & 1 & 0.95 & 0.92 & 0.89 & 0.96 \\
\hline
\end{tabular}

HepG2 cells were set up, grown and infected with $C$. trachomatis in presence or absence of mevastatin as described in Methods. RNA was extracted in 48 hours after inoculation of the bacteria. RNA levels for the genes of interest were normalized to 36B4 expression level, whose CT values are represented in the upper row of the Table. All RNA values in the infected cells are referenced to non-infected control. cholesterologenic enzymes (HMG-CoA reductase, HMG-CoA synthase, SS) which is well known effect of statins in the cultures cells [22]. Notably, LDL-receptor related protein mRNA was not impacted under all conditions studied.

\section{Mevastatin inhibits chlamydial growth in HepG2 cells}

Figure 1 shows representative immunofluorescent images of HepG2 cells infected with C. trachomatis in presence of increasing concentrations of mevastatin. As can be seen, the effect of mevastatin was marginal at the concentration of $1 \mu \mathrm{M}$, though some decline in the number of infected cells has been noticed. However, 20 $\mu \mathrm{M}$ mevastatin reduced both the number of inclusion bodies in the infected cells, promoting a perinuclear pattern of staining. Mevastatin-treated cells $(20 \mu \mathrm{M})$ appeared to contain smaller inclusion bodies similar to those that occur during persistent chlamydial infection [23]. The highest concentration of mevastatin tested (40 $\mu \mathrm{M})$ abolished the number of infected cells almost completely. Analysis of bacterial transcripts showed a similar tendency. As can be seen from Figure 2, 16S rRNA and euo mRNA were undetectable at highest concentration of mevastatin used, whereas at $20 \mu \mathrm{M}$ and $1 \mu \mathrm{M}$ mevastatin reduced the expression level for 16S rRNA by 8 and 3 fold respectively. There is significant induction of euo mRNA at $20 \mu \mathrm{M}$ mevastatin concentration.

Inhibition of chlamydial growth in cultured cells in presence of mevastatin may take place due to abnormal internalization of chlamydial particles, since the entry of chlamydial particles into mammalian cells requires interaction of pathogens with lipid rafts of plasma membrane [24]. Therefore, we next investigated the internalization rate of chlamydial particles into HepG2 cells in presence of $40 \mu \mathrm{M}$ mevastatin. As can be seen from Figure 3 , HepG2 cells treated with $40 \mu \mathrm{M}$ mevastatin have similar number of chlamydial particles attached to the plasma membrane when compared to untreated control cells. Mevastatin treatment did not affect the number of internalized particles as well (results not shown).

\section{Discussion}

Although there is a small but growing body of evidence that $C$. trachomatis can be disseminated widely throughout the human body, the physiological consequences and overall medical relevance of extragenital propagation of C. trachomatis remains poorly understood. First of all, our results confirm initial observations [25] showing the ability of $C$. trachomatis to propagate in HepG2 hepatoma cell line. More importantly, we have demonstrated that propagation of $C$. trachomatis in hepatocytes follows full infectious cycle leading to the formation of infectious progeny in 48 and 72 hours of post-infection period. Propagation of the pathogen 

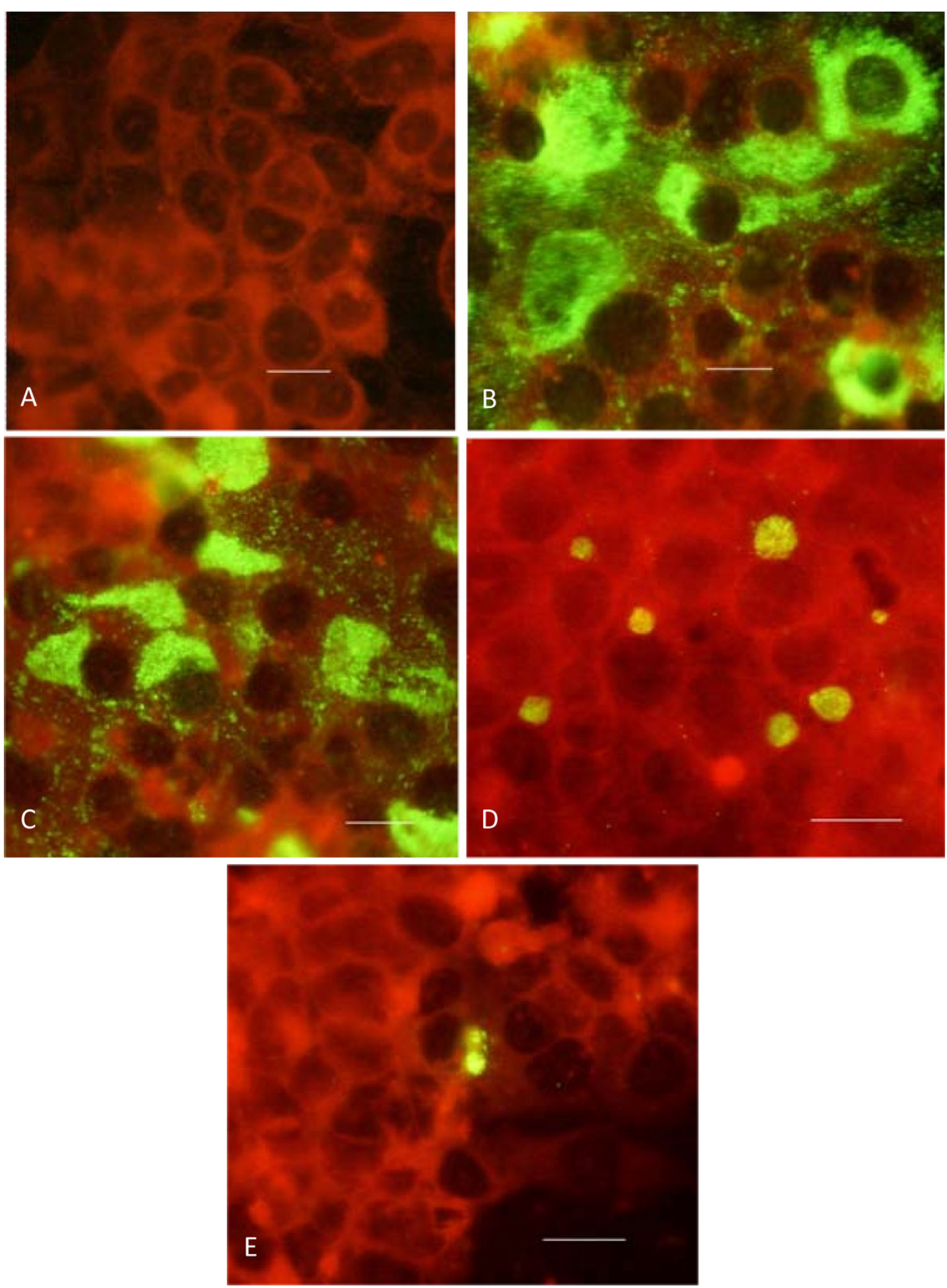

Figure 1 Immunofluorescent images of HepG2 cells infected with C. trachomatis in presence of mevastatin. HepG2 cells were set up, grown and infected with $\mathbf{C}$. trachomatis in presence or absence of mevastatin as described in Methods. Immunofluorescence analysis was performed 48 hours after inoculation of the pathogen. A - non-infected cells; $\mathbf{B}$ - infected cells with no mevastatin; $\mathbf{C}$ - infected cells in presence of $1 \mu \mathrm{M}$ mevastatin: D - infected cells in presence of $20 \mu \mathrm{M}$ mevastatin; $\mathbf{E}$ - infected cells in presence of $40 \mu \mathrm{M}$ mevastatin. Scale bar $=10 \mu \mathrm{m}$. 


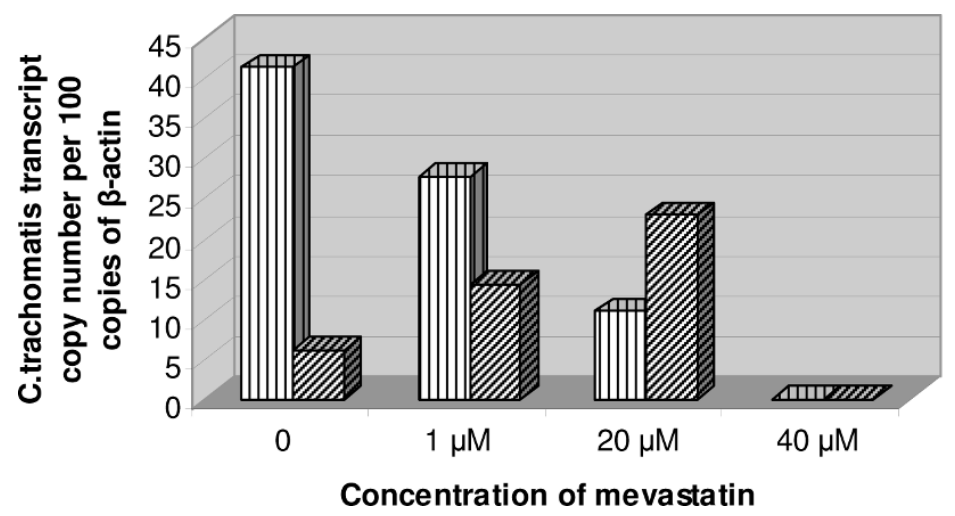

๓ 16S rRNA

reuo

Figure 2 Expression of chlamydial 16S RNA and euo in infected hepatocytes grown at different concentration of mevastatin. HepG2 cells were set up, grown and infected with C. trachomatis in presence or absence of mevastatin as described in Methods. RNA was extracted in 24 hours after inoculation of the bacteria. Expression of chlamydial genes was normalized to copy number of eukaryotic $\beta$-actin.

distinctively affects some specific functions of the liver cells. In particular, C.trachomatis ameliorates transcription of LDL-receptor in hepatocytes, which may have various consequences for lipid homeostasis.

Chlamydial organisms are strict intracellular parasites, whose requirements in the metabolites are covered by the host cells. Enhanced uptake of the substrates and metabolites by the infected host cells is a well known "signature" strategy of chlamydial infection mandatory for successful accomplishment of its infectious cycle [25]. However, in the case of the chlamydial growth in HepG2 cells we have seen significant decline in LDLreceptor mRNA, which may potentially result in the reduction of lipid uptake. The biological significance of this finding remains unclear. However it is possible to assume, that decline in the LDL-receptor mRNA might represent a mechanism of metabolic adaption of the host cell to chlamydial infection targeted on limitation of lipid supply and chlamydial growth in the cells. Unfortunately we were not able to document corresponding changes in LDL-receptor protein level due to decline in number of viable HepG2 cells that occurs at 72 hour time point of post-infection period. Models of persistent chlamydial infection might be required for evaluating hepatic LDL-receptor turnover in the infected liver cells.

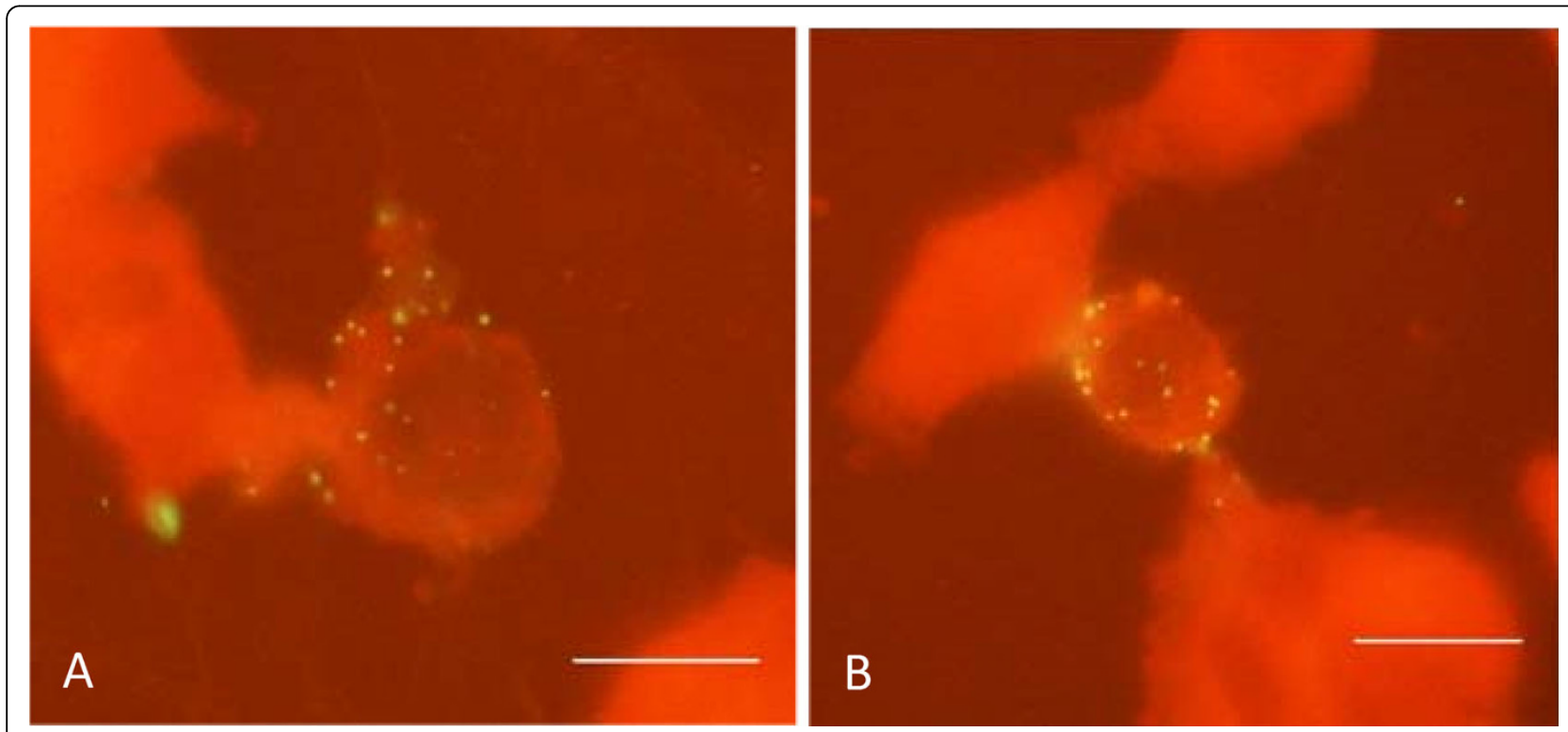

Figure 3 Attachment of chlamydial particles to plasma membrane of hepatocytes in presence or absence of mevastatin. HepG2 cells were set up, grown and incubated with chlamydial particles (EB) in presence or absence of mevastatin as described in Methods. Attached particles were visualized with FITC-labeled antibody against chlamydial LPS. A - attachment of chlamydial particles in absence of $40 \mu \mathrm{M}$ mevastatin: B - attachment of chlamydial particles in presence of $40 \mu \mathrm{M}$ mevastatin. Scale bar $=10 \mu \mathrm{m}$. 
Secondly, we have clearly shown that mevastatin, an inhibitor of cholesterol biosynthesis, restores LDL-receptor mRNA and has a significant anti-chlamydial activity reducing chlamydial growth in infected hepatocytes. Genome of C. trachomatis does not contain genes responsible for lipid biosynthesis. Chlamydial species are known to acquire cholesterol, fatty acids and triglycerides from the host cells [26]. Therefore, it was reasonable to believe that targeting the cholesterol biosynthetic pathway in the host cells might affect chlamydial infection rate. This prediction was confirmed by RT PCR analysis. It is well acknowledged, that $C$. trachomatis 16S rRNA gene expression is an informative criterion of chlamydial developmental cycle expressed in both early and late stages of C. trachomatis infection [27]. Detection of 16S rRNA transcript as a marker of viable and metabolically active Chlamydia allows to evaluate the effectiveness of different antibacterial agents [28]. Maximum inhibition of $16 \mathrm{~S}$ rRNA as well as drastic reduction in the number of infected immunofluorescencepositive cell has been seen at $40 \mu \mathrm{M}$ mevastatin level. Less pronounced decline in 16S rRNA transcript level has been observed at $20 \mu \mathrm{M}$ mevastatin concentration. Even though addition of $20 \mu \mathrm{M}$ mevastatin did not result in complete inhibition of chlamydial growth in HepG2 cells, there was formation of smaller chlamydial inclusions. Those are often observed in antibiotic- and/ or cytokine-treated cells when concentration of the agent is not enough to induce complete eradication of the pathogen [23]. "Aberrant" chlamydial cells are known to have some metabolic activity but fail to induce new rounds of chlamydial infection [23,28]. Therefore inhibition of chlamydial growth in mevastatin-treated HepG2 cells takes place in clearly dosedependent manner. Step-wise decline in 16S rRNA level was accompanied by reduction in the number of infected cells ( 1 and $20 \mu \mathrm{M}$ mevastatin), as well as the appearance of "aberrant" chlamydial forms $(20 \mu \mathrm{M}$ mevastatin) until complete eradication of chlamydial growth takes place $(40 \mu \mathrm{M}$ mevastatin). Euo mRNA level has been changing in a similar manner, except inconsistent increase seen at $20 \mu \mathrm{M}$ concentration of mevastatin. However, it is known that euo mRNA can be highly induced when the developmental cycle of $C$. trachomatis in cultured cells is compromised by addition of cytokines and other substances affecting chlamydial growth [28]. It has been proposed, that increased expression of euo may inhibit transcription of the genes specific for "late phase" of chlamydial developmental cycle $[28,29]$. Thus, enhanced transcription rate of euo may represent self-sufficient mechanism predetermining anti-chlamydial activity of mevastatin.

It is also important to conclude, that according to our results mevastatin has no effect on initial interaction of chlamydial particles with host cell, allowing the entry of the pathogen into hepatocytes. Therefore we assume that later stages of chlamydial developmental cycle are affected by mevastatin treatment. The effect of different metabolites and inhibitors of mevalonate pathway needs to be tested in hepatocytes infected with C. trachomatis in presence of mevastatin. It is possible, that anti-chlamydial activity of mevastatin takes place due to reduced geranylgeranylation of host cell proteins as it happens in case of lovastatin-treated hepatocytes infected with hepatitis $\mathrm{C}$ virus [30].

\section{Conclusions}

We have demonstrated that ongoing cholesterol synthesis is essential for chlamydial growth in hepatocytes. Although the precise mechanism of anti-chlamydial activity of mevastatin remains to be elucidated, targeting the cholesterol biosynthetic pathway may represent an effective strategy in management of chlamydial infection.

\section{Acknowledgements}

Ms Agni Roce is appreciated for invaluable help during experimental work and manuscript preparation.

\section{Author details}

${ }^{1}$ Cambridge Theranostics Ltd, Babraham Research Campus, Babraham, Cambridge, CB2 4AT, UK. ${ }^{2}$ Department of Medical Microbiology, Institute of Epidemiology and Microbiology RAMS, 18 Gamaleya Str, Moscow 123098, Russia.

\section{Authors' contributions}

YKB and NAZ contributed equally into design, acquisition of data, analysis and interpretation of the results. YPP and LNK performed immunostaining and RNA protocols. IMP contributed into primary concept, drafting the manuscript, and final approval for publishing the results. All authors read and approved the final manuscript.

\section{Competing interests}

The authors declare that they have no competing interests.

Received: 1 July 2009

Accepted: 28 January 2010 Published: 28 January 2010

\section{References}

1. Baguley S, Greenhouse P: Non-genital manifestations of Chlamydia trachomatis. Clinical Medicine 2003, 3:206-208.

2. Yang JL, Hong KC, Schachter J, Moncada J, Lekew T, House Jl, Zhou Z, Neuwelt MD, Rutar T, Halfpenny C, Shah N, Whitcher JP, Lietman TM: Detection of Chlamydia trachomatis ocular infection in trachomaendemic communities by rRNA amplification. Invest Ophthalmol Vis Sci 2009, 50:90-94.

3. Kobayashi $S$, Kida I: Reactive arthritis: recent advances and clinical manifestations. Intern Med 2005, 44:408-412.

4. Bilenki L, Wang S, Yang J, Fan Y, Joyee AG, Yang X: Chlamydia trachomatis NK T cell activation promotes infection in vivo. J Immunol 2005, 175:3197-3206.

5. Roubalova K: A simultaneous detection of Chlamydia pneumoniae and Chlamydia trachomatis DNA by real-time PCR. Epidemiol Mikrobiol Imunol 2007, 56:166-173.

6. Nasution TA, Cheong SF, Lim CT, Leong EW, Ngeow YF: Multiplex PCR for the detection of urogenital pathogens in mothers and newborns. Malays J Pathol 2007, 29:19-24.

7. Schrader S, Klos A, Hess S, Zeidler H, Kuipers JG, Rihl M: Expression of inflammatory host genes in Chlamydia trachomatis-infected human monocytes. Arthritis Res Ther 2007, 9:R54. 
8. Dreses-Werringloer U, Gérard HC, Whittum-Hudson JA, Hudson AP: Chlamydophila (Chlamydia) pneumoniae infection of human astrocytes and microglia in culture displays an active, rather than a persistent, phenotype. Am J Med Sci 2006, 332:168-174.

9. Yang X, Coriolan D, Schultz K, Golenbock DT, Beasley D: Toll-like receptor 2 mediates persistent chemokine release by Chlamydia pneumoniaeinfected vascular smooth muscle cells. Arterioscler Thromb Vasc Biol 2005, 25:2308-2314.

10. Wang G, Burczynski F, Hasinoff B, Zhong G: Infection of myocytes with Chlamydiae. Microbiology 2002, 148:3955-3959.

11. Rihl M, Köhler L, Klos A, Zeidler H: Persistent infection of Chlamydia in reactive arthritis. Ann Rheum Dis 2006, 65:281-284.

12. Shabot JM, Roak GD, Truant AL: Chlamydia trachomatis in the ascitic fluids of patients with chronic liver disease. Am J Gastroenterol 1983, 78:291-294.

13. Shabot AM: Chlamydia trachomatis and ascites: Going with the flow? Hepatology 2005, 9:505-506.

14. Dan M, Tyrrell LDJ, Goldsand G: Isolation of Chlamydia trachomatis from the liver of patients with prolonged fever. Gut 1987, 28:1514-1516.

15. Chen CJ, Wu KG, Tang RB, Yuan HC, Soong WJ, Hwang BT: Characteristics of Chlamydia trachomatis infection in hospitalized infants with lower respiratory tract infection. J Microbiol Immunol Infect 2007, 40:255-259.

16. Barteneva $\mathrm{N}$, Theodor I, Peterson EM, de la Maza LM: Role of neutrophils in controlling early stages of a Chlamydia trachomatis infection. Infect Immun 1996, 64:4830-4833.

17. Hatch GM, McClarty G: C.trachomatis-infection accelerates metabolism of phosphatidylcholinederived from low density lipoproteins but does not affect phosphatidylcholine secretion from hepatocytes. BMC Microbiology 2004, 4:8.

18. Wang G, Burczynski F, Anderson J, Zhong G: Effect of host fatty acidbinding protein and fatty acid uptake on growth of Chlamydia trachomatis L2. Microbiology 2007, 153:1935-1939.

19. Galdwell HD, Kromhout J, Schachter J: Purification and partial charachterization of the major outer membrane protein of Chlamydia trachomatis. Infect Immun 1981, 31:1161-1176.

20. Carabeo RA, Grieshaber SS, Fisher E, Hackstadt T: Chlamydia trachomatis induces remodeling of the actin cytoskeleton during attachment and entry into HeLa cells. Infect Immun 2002, 70:3793-3803.

21. Goldstein JL, Brown MS: The LDL receptor. Arterioscler Thromb Vasc Biol 2009, 29:431-8.

22. Medina MW, Gao F, Ruan W, Rotter Jl, Krauss RM: Alternative splicing of 3hydroxy-3-methylglutaryl coenzyme A reductase is associated with plasma low-density lipoprotein cholesterol response to simvastatin Circulation 2008, 118:355-362.

23. Hammerschlag MR: The intracellular life of chlamydiae. Semin Pediatr Infect Dis 2002, 13:239-248.

24. Stuart ES, Webley WC, Norkin LC: Lipid rafts, caveolae, caveolin-1, and entry by Chlamydiae into host cells. Exp Cell Res 2003, 287:67-78.

25. Wang G, Burczynski F, Anderson J, Zhong G: Effect of host fatty acidbinding protein and fatty acid uptake on growth of Chlamydia trachomatis L2. Microbiology 2007, 153:1935-1939.

26. Kumar Y, Cocchiaro J, Valdivia RH: The obligate intracellular pathogen Chlamydia trachomatis targets host lipid droplets. Curr Biol 2006, 16:1646-1651

27. Belland RJ, Zhong G, Crane DD, Hogan D, Sturdevant D, Sharma J, Beatty WL, Caldwell HD: Genomic transcriptional profiling of the developmental cycle of Chlamydia trachomatis. Proc Natl Acad Sci USA 2003, 100:8478-8483.

28. Perry LL, Su H, Feilzer K, Messer R, Hughes S, Whitmire W, Caldwell HD: Differential sensitivity of distinct Chlamydia trachomatis isolates to IFNgamma-mediated inhibition. J Immunol 1999, 162:3541-3548.

29. Zhang L, Douglas AL, Hatch TP: Characterization of a Chlamydia psittaci DNA binding protein (EUO) synthesized during the early and middle phases of the developmental cycle. Infect Immun 1998, 66:1167-1173.

30. Ye J, Wang C, Sumpter R Jr, Brown MS, Goldstein JL, Gale M Jr: Disruption of hepatitis $C$ virus RNA replication through inhibition of host protein geranylgeranylation. Proc Natl Acad Sci USA 2003, 100:15865-15870.

doi:10.1186/1476-5926-9-3

Cite this article as: Bashmakov et al: Chlamydia trachomatis growth inhibition and restoration of LDL-receptor level in HepG2 cells treated with mevastatin. Comparative Hepatology 2010 9:3.

\section{Submit your next manuscript to BioMed Central and take full advantage of:}

- Convenient online submission

- Thorough peer review

- No space constraints or color figure charges

- Immediate publication on acceptance

- Inclusion in PubMed, CAS, Scopus and Google Scholar

- Research which is freely available for redistribution

Submit your manuscript at www.biomedcentral.com/submit 